\title{
Study on Catalytic Performance of Heteropoly Acid Supported on MCM-41 Molecular Sieve in Synthesized of Butyl lactate
}

\author{
LING Xu ${ }^{1, a}$, HAI Yun Yu ${ }^{1, b}$, LI Mei Duan ${ }^{1, c}$ and ZONG Rui Liu ${ }^{1, d}$ \\ ${ }^{1}$ College of Chemistry and Chemical Engineering, Inner Mongolia \\ University for the Nationalities, Tongliao, China, 028000 \\ atlxuling1979@163.com, bthyy@163.com, duanImxie@126.com, 'liuzr@imun.edu.cn
}

Keywords: Heteroply acid. Molecular sieve. Butyl lactate. Synthesis.

\begin{abstract}
The supported heteropoly acid catalysts were prepared by loading Phosphotungstic acid on the MCM-41 molecular sieve via the impregnation method, which were characterized by FT-IR, N2 adsorption-desorption and applied in the synthesis of butyl lactate. The results show that heteropoly acids supported on porous molecular sieve, which can improve the catalytic performance of heteropoly acids, improve its catalytic activity and is good method to solve hard problem of recycling use.
\end{abstract}

\section{Introduction}

Heteropoly acid is a solid acid [1], which is applied as acid catalyst, redox catalyst and bifunctional catalyst in the chemical synthesis [2], shows high catalytic activity and selectivity, and has great advantages such as high reactivity, non-corrosion to equipments and no pollution. Consequently, it becomes the hot topic of research in catalysis. However, the application of heteropoly acid in the catalytic fields is limited because of its small specific surface area, poor stability, easily dispersing in polar solvents, difficulty of recycling and other reasons.

In this report, the supported heteropoly acid catalysts were prepared by loading Phosphotungstic acid on the MCM-41 molecular sieve via the impregnation method, which were applied in the synthesis of butyl lactate. The experiment results show that MCM-41 molecular sieve was an excellent carrier for heteropoly acid, and the supported heteropoly acid catalysts possessed high catalytic activity in synthesis of butyl lactate.

\section{Experiments}

The preparation of MCM-41 molecular sieve [3]. Typically, 1.10g cetyltrimethylammonium bromide (CTAB) was dissolved in $25 \mathrm{~mL}$ of distilled water, followed by adding $12 \mathrm{~mL}$ of $\mathrm{NH} 4 \mathrm{OH}$ (25\%). Then $5 \mathrm{~mL}$ of tetraethyl orthosilicate (TEOS) was slowly added to the resulting solution and kept stirring at room temperature for $3 \mathrm{~h}$. Next, the mixture was enclosed in reaction kettle for crystallization for $24 \mathrm{~h}$ at $373 \mathrm{~K}$. The products were filtrated, washed with distilled water repeatedly and dried. At last, it was baked at $823 \mathrm{~K}$ in air to remove template to obtain the products.

Preparation of the supported heteropoly acid catalysts in the impregnation method $[4,5]$. (0.43g, 0.67g, 1.0g) Phosphotungstic acid (HPW) was dissolved in $25 \mathrm{~mL}$ of double-distilled water. After completely dissolving, $1 \mathrm{~g}$ the above prepared MCM-41 molecular sieve was added into the resulting solution. And the resulting solution was stirred at $353 \mathrm{~K}$ until the supported heteropoly acid catalysts were obtained. Based on the loading of active component, the catalysts were named as 30\%HPW-MCM-41, 40\%HPW-MCM-41, 50\%HPW-MCM-41.

\section{Results and discussion}

FT-IR analysis. The FT-IR spectra images of HPW-MCM-41 samples that supported Phosphotungstic acid are shown in Fig.1. It can be seen clearly from Fig.1-a that the absorbance bond at 465 and $800 \mathrm{~cm}^{-1}$ is due to flexural vibration and symmetric stretching vibration of Si-O-Si, and 
the band at $1080 \mathrm{~cm}-1$ is attributed to the asymmetrical stretching vibrations of the Si-O-Si bond. The peaks at ca. $3450 \mathrm{~cm}^{-1}$ are related to the absorbance bond of $\mathrm{OH}$, suggesting that there are massive hydroxyl groups on the surface of molecular sieve. The FT-IR images (Fig. 1-b, c) of the supported heteropoly acid catalysts indicate that the absorption peak in the region $800 \sim 1000 \mathrm{~cm}-1$ was extremely weak but very broad. With the increase of the loading, the characteristic absorption peak of Phosphotungstic acid emerges clearly (Fig. 1-d), which shows that Phosphotungstic acid could combine with support very well to form strong peaks.

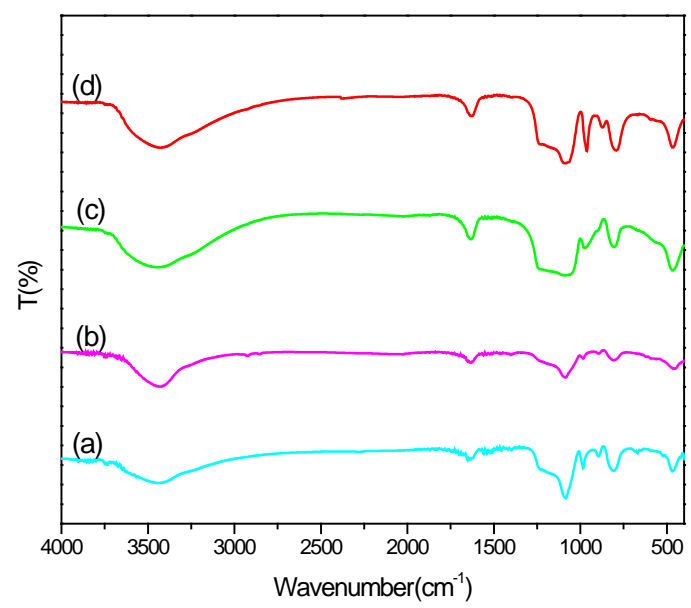

Fig.1 FT-IR spectra of HPW-MCM-41 series catalyst

(a) MCM-41, (b)30\%-HPW-MCM-41, (c) 40\%-HPW-MCM-41, (d) 50\%-HPW-MCM-41

N2 adsorption-desorption results. Fig 2 (A) presents the nitrogen adsorption-desorption isotherms of a series of HPW-MCM-41 samples at 77K. As can be seen from the Fig. 2 (A), the hysteresis loop appears at $\mathrm{P} / \mathrm{P} 0=0.5 \sim 0.9$ and the isotherms are of type IV according to the BDDT classification, which is the characteristic of mesoporous materials. With increasing the loading of samples, the adsorption capacities of nitrogen becomes less and less, and the hysteresis loop shifts toward to type $\mathrm{H} 2$. If there is capillary condensation when the value of $\mathrm{P} / \mathrm{P} 0$ is 0.4 , we can conclude that the materials are mesoporous structure. So with variation of relative pressures, the sharp capillary condensation in the isotherms is associated with orderly mesoporous opening structure. In addition, no damage or destruction occurs in the channels with the loading of samples increasing.
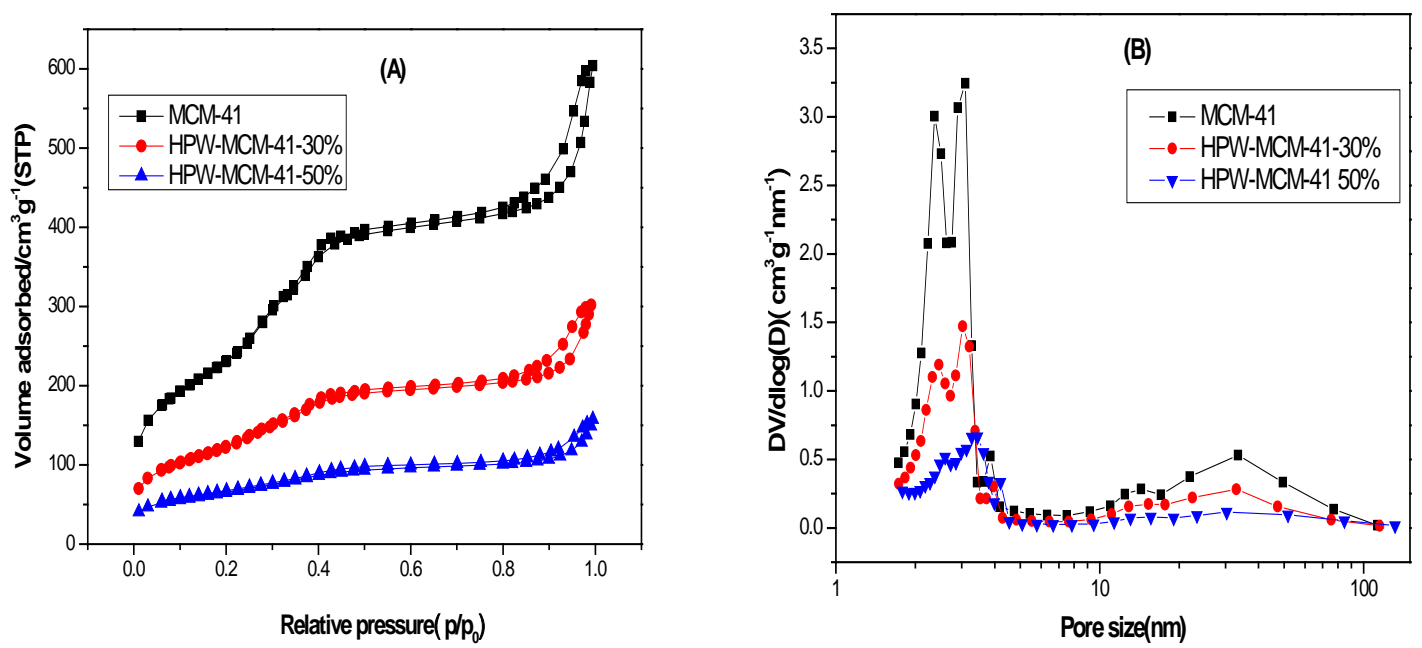

Fig.2 $\mathrm{N}_{2}$ adsorption-desorption isotherms (A) and pore size distribution (B) of HPM-MCM-41 catalyst 
The pore size distribution of the novel materials are shown in Fig. 2 (B). Increasing the loadings of Phosphotungstic acid results in decreasing of the pore size and diameter, which suggests that Phosphotungstic acid entered into the channels. Meanwhile we found that the pore size decreases when the loading further increases.

\section{Research on catalytic activity}

Synthesis processes of butyl lactate [6]. Three-necked flask is equipped with Allihn condenser, thermometer and water separator. The certain molar ratios of lactic acid, n-Butyl alcohol, and the supported heteropoly acid were added into three-necked flask. The constant temperature heating jacket was used to control temperature, stir and heat. After a period of time, the reaction temperature was allowed to cool down to ambient temperature. Then the reaction solution was separated from the catalyst by filtering using vacuum circulating water pump and washed with saturated sodium carbonate solution and distilled water. Dried with anhydrous magnesium sulfate, the mixture was transferred into round bottomed flask to distill. We collected the fraction at 433 453K and removed the unreacted n-Butyl alcohol. The former distilled fraction (371 33K) was twice-distilled and collected the fraction(433 453K). The two fractions were combined. Meanwhile, the separated catalysts were recycled after drying and activating.

Evaluation index. Esterification rate: the results are measured according to GB/T 1668-2008 [7], which use $\mathrm{KOH}-\mathrm{CH} 3 \mathrm{CH} 2 \mathrm{OH}$ solution titrant to titrate acid number to achieve esterification rate.

$\mathrm{R}=(1-\mathrm{W} 2 / \mathrm{W} 1) \times 100 \%$, where $\mathrm{R}$ is esterification rate, W1and $\mathrm{W} 2$ are acid value before and after the experiments.

\section{The performance of the supported heteropoly acids catalysts [8]}

Determination quantities of the supported catalysts. We take 50\%-HPW-MCM-41 catalyst as an example to determine quantities of catalyst effecting esterification rate. The results are shown in Fig. 3.

Esterification is an autocatalytic reaction. Consequently the rate can reach $40 \%$ without catalyst. Adding 50\%-HPW-MCM-41 samples, the rate increases rapidly. When the addition reaches $2.5 \mathrm{~g} 3.0 \mathrm{~g}$, esterification rate doesn’t varies obviously, so we choose $1.5 \mathrm{~g}$ catalysts as the best value.

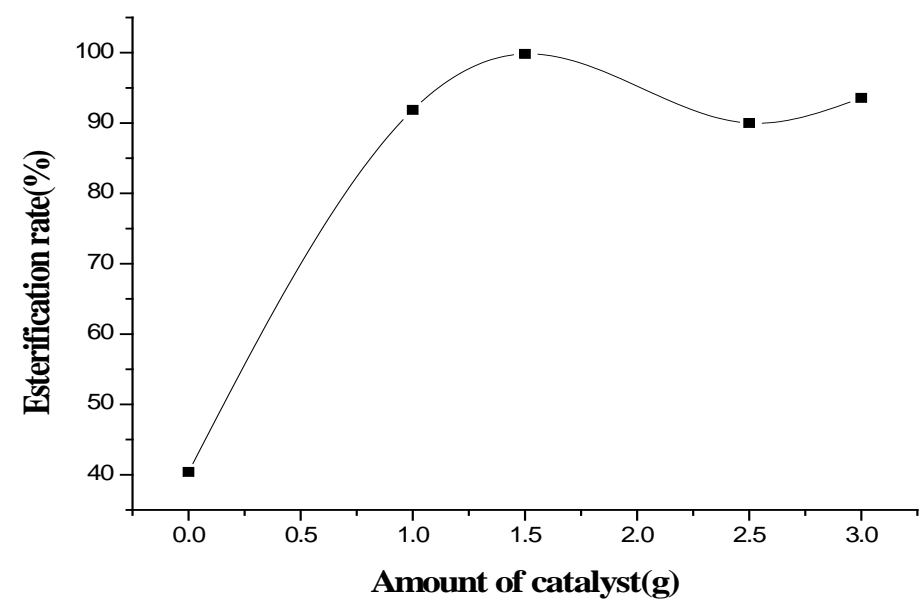

Fig. 3 Effect on esterification rate at different amount of catalyst

Recycling utilization of HPW-MCM-41 series catalysts. To check out the lifetime of the catalysts, we heat the catalysts at 353K to make it activated and then to use in esterification reaction after the separation between them and reaction solution. Summary of the esterification rates after the catalysts recycling in 5 times is listed in Table 1. 
Table 1 Recycling utilization of HPW-MCM-41 series catalyst

\begin{tabular}{cccccc}
\hline Times & 1 & 2 & 3 & 4 & 5 \\
\hline Esterification rate (\%) & 98.49 & 82.28 & 79.84 & 74.36 & 72.11 \\
\hline
\end{tabular}

\section{Summary}

(1) The supported heteropoly acid catalysts HPW-MCM-41 show good catalytic activity and high selectivity. Especially, a series of HPW-MCM-41 exhibits over 98\% esterification rate.

(2) The catalysts could be recycled by a simple process, including the separating of catalysts and reaction liquids, activating of catalysts and reusing. However, the performance of the catalysts decreases in the recycle process. This mainly depends on the tightness of the interaction between heteropoly acid and the surface of the carrier, and the dissolution problems of heteropoly acid on supports in the liquid phase reaction. Part of heteropoly acid dissolves in the reaction, resulting in decreasing of the catalytic efficiency.

\section{Acknowledgments}

We acknowledge the financial support of the National Natural Science Foundation of China (NO. 21261014), the Natural Science Foundation of Inner Mongolia (2012MS0207), and Tongliao city and Inner Mongolia University for the Nationalities cooperation projects in science and technology (SXYB2012052).

\section{References}

[1] Enbo Wang, Changwen Hu, Lin Xu: Multi acid chemistry (Chemical Industry Press, Beijing, 1998).

[2] Enbo Wang, Yangguang Li, Ying Lu: Multi acid chemistry introduction (Northeast Normal University Press, Changchun 2009)..

[3] Tao Tao: The Research of Synthesis and Catalytic Performance of MCM-41 Mesoporous Molecular Sieves (Master Dissertation of Jiangsu University, 2006).

[4] Ruren $\mathrm{Xu}$, Wenqin Pang: Chemistry of zeolites and porous materials (Science Press, Benjing 2004).

[5] Shaopeng Wang: Preparation of Heteropoly Acid Supported on Molecular Sieve SBA-15 and Study on Catalysis Synthesis of Tributly Citrate (Master Dissertation of Taiyuan University of Technology, 2004).

[6] Yingjun Zhang and Haijun Cheng: China Molybdenum Industry Vol. 33 (2009), p. 27.

[7] Yanping Guo and Xiuli Fan, China Patent GB/T1668-2008. (2008).

[8] Wenai Han: Hebei Chemical Engineering and Industry Vol. 31 (2008), p. 31. 